\title{
CRESCIMENTO INICIAL DE ESPÉCIES FLORESTAIS DE DIFERENTES GRUPOS SUCESSIONAIS EM RESPOSTA A DOSES DE FÓSFORO'
}

\author{
ÁLVARO VILELA DE RESENDE², ANTONIO EDUARDO FURTINI NETO \\ JOELAUGUSTO MUNIZ ${ }^{4}$, NILTON CURI ${ }^{3}$ e VALDEMAR FAQUIN ${ }^{3}$
}

\begin{abstract}
RESUMO - Objetivando avaliar a resposta de espécies florestais ao fornecimento de $\mathrm{P}$, conduziu-se um ensaio sob condições de casa de vegetação, cultivando-se mudas das espécies arbóreas pioneiras (aroeira - Lithraea molleoides; aroeirinha - Schinus terebinthifolius; jacaré - Piptadenia gonoacantha; sabiá - Mimosa caesalpiniaefolia; sesbânia - Sesbania virgata), clímax exigente em luz (jatobá - Hymenaea courbaril), e clímax tolerantes a sombra (guanandi - Calophyllum brasiliensis; ipê-amarelo - Tabebuia serratifolia; óleo-bálsamo - Myroxylon peruiferum). Utilizaram-se cinco doses de $\mathrm{P}$, correspondentes a $0,100,250,500$ e $800 \mathrm{mg} \mathrm{dm}^{-3} \mathrm{de}$ P. Foram avaliados o diâmetro do caule, a altura e a matéria seca de raízes, parte aérea e total das plantas. As espécies pioneiras foram mais responsivas ao fornecimento de $\mathrm{P}$, indicando a necessidade do suprimento deste nutriente para o adequado desenvolvimento destas espécies. As espécies clímax mostraram-se pouco sensíveis ao suprimento de $\mathrm{P}$, refletindo um baixo requerimento na fase de mudas. Diferenças em relação à taxa de crescimento e ao tamanho das sementes podem estar ligadas ao comportamento contrastante observado para espécies pioneiras e clímax.
\end{abstract}

Termos para indexação: grupos ecológicos, crescimento de mudas, adubação fosfatada.

\section{INITIAL GROWTH OF FOREST SPECIES OF DIFFERENT SUCCESSIONAL GROUPS IN RESPONSE TO PHOSPHORUS DOSES}

\begin{abstract}
With the aim of evaluating the responses of forest species to phosphorus supply, an assay under greenhouse conditions was carried out, where seedlings of pioneer tree species (Lithraea molleoides, Schinus terebinthifolius, Piptadenia gonoacantha, Mimosa caesalpiniaefolia, Sesbania virgata), a light-demander climax species (Hymenaea courbaril), and the shade-tolerant climaxes species (Calophyllum brasiliensis, Tabebuia serratifolia, Myroxylon peruiferum) were cultivated. Five phosphorus doses were used, corresponding to $0,100,250,500$ and $800 \mathrm{mg} \mathrm{dm}^{-3}$ of P. Stem diameter, height, and root, shoot and total dry matter yield of the plants were evaluated. The pioneers species were more responsive to phosphorus furnishing, indicating the need of phosphorus supply for the adequate development of these species. The climaxes species showed to have low sensitivity to phosphorus supply, reflecting a lower requirement in the initial growth period. The differences in relation to growth rate and seed size may be connected to the contrasting behavior observed for pioneers and climaxes species.
\end{abstract}

Index terms: ecologic groups, seedling growth, phosphated fertilization.

${ }^{1}$ Aceito para publicação em 4 de fevereiro de 1999.

Extraído da Dissertação de Mestrado, apresentada pelo primeiro autor à UFLA. Trabalho financiado pela CAPES, FAPEMIG e CEMIG

${ }^{2}$ Eng. Agr., M.Sc., Dep. de Ciências Agrárias, Universidade Estadual de Montes Claros (UNIMONTES), Caixa Postal 91, CEP 39440-000 Janaúba, MG.

${ }^{3}$ Eng. Agr., Dr., Dep. de Ciência do Solo, Universidade Federal de Lavras (UFLA), Caixa Postal 37, CEP 37200-000 Lavras, MG. E-mail: afurtini@ufla.br

${ }^{4}$ Eng. Agr., Dr., Dep. de Ciências Exatas, UFLA.

\section{INTRODUÇÃO}

Em virtude da dinâmica do $\mathrm{P}$ nos solos mais intemperizados, a sua baixa disponibilidade para as plantas tem sido apontada como causa do inadequado desenvolvimento da maioria das culturas em solos das regiões tropicais. Nessas áreas, onde os solos possuem elevada capacidade de fixação, a deficiência do nutriente é o mais importante fator 
nutricional a restringir o crescimento vegetal (Sanchez \& Salinas, 1981)

Uma estratégia a ser buscada é a obtenção de genótipos mais eficientes em se desenvolver sob condições adversas de ambiente, como é o caso das condições nutricionais restritivas dos solos que predominam em grande parte do território brasileiro e em especial nas áreas destinadas ao plantio de espécies florestais para recuperação e conservação ambiental.

Embora os trabalhos envolvendo respostas ao fornecimento de $\mathrm{P}$ pelas espécies empregadas em florestamento e reflorestamento para fins ambientais sejam escassos, têm sido observadas respostas à adubação fosfatada em solos deficientes no nutriente. A adubação de plantio com NPK como forma de garantir o estabelecimento inicial das mudas no campo é, dessa forma, recomendável, apesar de as espécies apresentarem diferentes demandas por $\mathrm{P}$ (Siqueira et al., 1995).

Em geral, nesses estudos têm sido encontradas respostas de espécies florestais a doses moderadas de P (Faria et al., 1995, 1996; Lima et al., 1996; Paron et al., 1996), havendo casos até de redução de crescimento sob adubações mais pesadas (Lima, 1995; Rocha, 1995; Faria et al., 1996), evidenciando um baixo requerimento do nutriente em algumas espécies.

De modo geral, as espécies pioneiras têm seu potencial de crescimento mais restringido quando se desenvolvem em solos pobres, mostrando-se bastante responsivas à fertilização, ao passo que, com o avanço do grupo sucessional, o estímulo ao crescimento proporcionado pela adubação é menos pronunciado e algumas vezes inexistente; tendência, em parte, atribuída ao crescimento mais lento, característico das espécies clímax. Conforme Marschner (1991) e Lambers \& Poorter (1992), espécies de crescimento lento apresentam baixa resposta ao fornecimento de nutrientes; também uma característica de adaptação a solos pouco férteis.

A elevada resposta de algumas espécies à melhoria da fertilidade do solo pode então ser devida à sua maior taxa de crescimento, requerendo, deste modo, maior quantidade de nutrientes para atender à demanda nutricional, o que, em última análise, permite a expressão do potencial de produ- ção de biomassa das espécies de crescimento inicial mais acentuado (Silva et al., 1997). Esses autores sugerem que as maiores respostas podem ser observadas em espécies com maiores taxas de crescimento, independentemente do grupo sucessional.

Objetivou-se no presente estudo avaliar o comportamento de espécies florestais de diferentes grupos sucessionais quanto ao crescimento vegetativo inicial, em resposta a doses de $\mathrm{P}$.

\section{MATERIAL E MÉTODOS}

Foi conduzido um ensaio em casa de vegetação, no Departamento de Ciência do Solo da Universidade Federal de Lavras (UFLA), utilizando-se amostra da camada superficial $(0-20 \mathrm{~cm})$ de um Latossolo Vermelho-Escuro, distrófico, argiloso, fase cerrado tropical subcaducifólio, relevo suave ondulado (Chagas, 1994), proveniente do distrito de São Sebastião da Vitória, MG, na área sob influência dos reservatórios das hidrelétricas da CEMIG de Camargos/Itutinga, MG.

Para aplicação dos tratamentos, foi realizada a correção da acidez, na qual se utilizou uma mistura de $\mathrm{CaCO}_{3}$ + $\mathrm{MgCO}_{3}$ na relação $\mathrm{Ca}: \mathrm{Mg}$ de 4:1, em dosagem baseada no método das curvas de incubação, para se atingir $\mathrm{pH}$ em torno de 6,0. Foram empregados tratamentos com o fornecimento de cinco doses de $\mathrm{P}$, correspondentes à aplicação de $0,100,250,500$, e $800 \mathrm{mg} \mathrm{dm}^{-3} \mathrm{de}$ P. Foi aplicada, em todas as parcelas, uma fertilização básica com $100 \mathrm{mg}$ de $\mathrm{N}, 100 \mathrm{mg}$ de $\mathrm{K}, 45 \mathrm{mg}$ de $\mathrm{S}, 0,5 \mathrm{mg}$ de $\mathrm{B}, 1,5 \mathrm{mg}$ de $\mathrm{Cu}, 3,6 \mathrm{mg}$ de $\mathrm{Mn}, 0,15 \mathrm{mg}$ de Mo, e 5,0 mg de Zn por $\mathrm{dm}^{3}$ de solo. $\mathrm{O}$ fornecimento de $\mathrm{P}$ e a fertilização básica foram efetuados via sais p.a., e balanceados de maneira que apenas a quantidade de $\mathrm{P}$ variou, permanecendo os demais nutrientes nivelados em todos os tratamentos. A correção, os tratamentos e as fertilizações, foram realizados individualmente, em cada vaso com capacidade para três $\mathrm{dm}^{3} \mathrm{de}$ substrato.

Precedendo o transplantio das espécies, os vasos contendo solo com os corretivos e fertilizantes foram umedecidos a $60 \%$ do volume total de poros, e incubados durante 20 dias. Após este período, foram retiradas amostras de solo para análises químicas visando caracterizar o efeito dos tratamentos (Tabela 1). As análises foram realizadas nos laboratórios do Departamento de Ciência do Solo/UFLA, com base nos métodos de Vettori (1969), com modificações feitas pela Embrapa (1979): $\mathrm{pH}$ em água na relação 1:2,5, P e K pelo extrator Mehlich-1, e Ca, Mg e $\mathrm{Al}$ por extração com $\mathrm{KCl} 1 \mathrm{~N}$. 
Foram estudadas nove espécies florestais arbóreas, bem definidas quanto ao grupo sucessional (Tabela 2), cujas sementes foram coletadas de plantas matrizes na região de Lavras, $\mathrm{MG}$, onde o clima é de transição entre Cwb e Cwa, segundo Köppen, com temperatura média anual de $19,4^{\circ} \mathrm{C}$ e altitude de $910 \mathrm{~m}$. As sementes foram postas a germinar em bandejas contendo vermiculita expandida. Após a emergência das plântulas, procedeu-se ao transplantio para o solo com os tratamentos, conduzindose duas plantas por vaso.

TABELA 1. Características químicas do material de solo após correção e aplicação dos tratamentos.

\begin{tabular}{lrrrrr}
\hline Características & \multicolumn{5}{c}{ Doses de fósforo $\left(\mathrm{mg} \mathrm{dm}^{-3}\right)$} \\
\cline { 2 - 6 } & 0 & 100 & 250 & 500 & 800 \\
\hline $\mathrm{pH}\left(\mathrm{H}_{2} \mathrm{O}\right)$ & 6,0 & 6,0 & 6,0 & 6,1 & 6,1 \\
$\mathrm{P}\left(\mathrm{mg} \mathrm{dm}^{-3}\right)$ & 1 & 3 & 9 & 29 & 67 \\
$\mathrm{~K}\left(\mathrm{mg} \mathrm{dm}^{-3}\right)$ & 150 & 154 & 147 & 154 & 151 \\
$\mathrm{Ca}\left(\mathrm{mmol}_{\mathrm{c}} \mathrm{dm}^{-3}\right)$ & 30 & 29 & 26 & 27 & 25 \\
$\mathrm{Mg}\left(\mathrm{mmol}_{\mathrm{c}} \mathrm{dm}^{-3}\right)$ & 6 & 4 & 6 & 9 & 8 \\
$\mathrm{Al}\left(\mathrm{mmol}_{\mathrm{c}} \mathrm{dm}^{-3}\right)$ & 0 & 0 & 0 & 0 & 0 \\
$\mathrm{H}+\mathrm{Al}\left(\mathrm{mmol}_{\mathrm{c}} \mathrm{dm}^{-3}\right)$ & 29 & 29 & 32 & 36 & 40 \\
$\mathrm{~S}\left(\mathrm{mmol}_{\mathrm{c}} \mathrm{dm}^{-3}\right)$ & 40 & 37 & 36 & 40 & 37 \\
$\mathrm{~m}(\%)$ & 0 & 0 & 0 & 0 & 0 \\
$\mathrm{~V}(\%)$ & 58 & 56 & 53 & 53 & 48 \\
\hline
\end{tabular}

A umidade do solo foi mantida em torno de $60 \%$ do volume total de poros (Freire et al., 1980) por meio de irrigações diárias com água deionizada. Três adubações complementares com $\mathrm{N}$ em cobertura, na forma de $\mathrm{NH}_{4} \mathrm{NO}_{3}$, foram efetuadas no decorrer do experimento, totalizando um fornecimento suplementar de 45 e $70 \mathrm{mg} \mathrm{dm}^{-3} \mathrm{deN}$ na dose 0 de $\mathrm{P}$ e nas demais doses, respectivamente.

Aos 90 dias após o transplantio, foram avaliados o diâmetro do caule na região do colo e a altura das plantas, que foram cortadas rente ao coleto e o material vegetal separado em raízes, caules e folhas e secado em estufa de circulação forçada de ar a $60^{\circ} \mathrm{C}$ até peso constante, para determinação da matéria seca das espécies nos diversos tratamentos.

O delineamento experimental adotado foi o inteiramente casualizado, com cinco repetições, e os tratamentos constituíram um fatorial completo com as nove espécies e as cinco doses de $\mathrm{P}$. Os dados foram submetidos à análise de variância, em que procurou-se isolar o comportamento das espécies em resposta às doses de $\mathrm{P}$, analisando-as em grupos individualizados de espécies pioneiras e clímax. Foram ajustadas equações de regressão para o crescimento em altura e diâmetro do caule, produção de matéria seca de raizes (MSR), parte aérea (caules + folhas) (MSPA) e total (raízes + parte aérea) (MST), e relação raiz-parte aérea (RELRP), como variáveis dependentes das doses de P. A partir das equações foram calculadas, as doses de $\mathrm{P}$, correspondentes a $90 \%$ do máximo crescimento em altura, diâmetro do caule e produção de matéria seca de cada espécie, onde, no caso do modelo ajustado ser o linear, adotou-se a maior dose aplicada $\left(800 \mathrm{mg} \mathrm{dm}^{-3}\right)$.

TABELA 2. Caracterização das espécies florestais estudadas.

\begin{tabular}{|c|c|c|c|c|}
\hline Nome comum & Nome científico & Família & $\begin{array}{c}\text { Grupo } \\
\text { ecológico }\end{array}$ & $\begin{array}{c}\text { Sementes } \\
\left(\mathrm{n}^{\mathrm{o}} \mathrm{kg}^{-1}\right)\end{array}$ \\
\hline Aroeira & Lithraea molleoides (Vell.) Engl. & Anacardiaceae & $\mathrm{P}$ & 31.500 \\
\hline Aroeirinha & Schinus terebinthifolius Raddi & Anacardiaceae & $\mathrm{P}$ & 68.700 \\
\hline Jacaré & Piptadenia gonoacantha (Mart.) Macbr. & Mimosaceae & $\mathrm{P}$ & 16.200 \\
\hline Sabiá & Mimosa caesalpiniaefolia Benth. & Mimosaceae & $\mathrm{P}$ & 41.000 \\
\hline Sesbânia & Sesbania virgata Merril. & Fabaceae & $\mathrm{P}$ & 13.000 \\
\hline Guanandi & Calophyllum brasiliensis Camb. & Clusiaceae & Cs & 625 \\
\hline Ipê-amarelo & Tabebuia serratifolia (Vahl) Nich. & Bignoniaceae & Cs & 14.900 \\
\hline Jatobá & Hymenaea courbaril L. & Caesalpiniaceae & $\mathrm{Cl}$ & 210 \\
\hline Óleo-bálsamo & Myroxylon peruiferum L.f. & Fabaceae & Cs & 1.580 \\
\hline
\end{tabular}

Fonte: Davide et al. (1995) 


\section{RESULTADOS E DISCUSSÃO}

\section{Crescimento e produção de matéria seca}

A aplicação de $P$ provocou sensíveis mudanças nos níveis de disponibilidade do nutriente, enquanto as demais características químicas do solo, em todos os tratamentos, podem ser consideradas como satisfatórias para o desenvolvimento de espécies arbóreas em geral (Tabela 1).

As quantidades relativamente pequenas de $\mathrm{P}$, recuperadas após a incubação do solo com os tratamentos, se devem à forte fixação do elemento pelo solo utilizado no experimento, o qual apresenta uma adsorção máxima de fosfatos da ordem de $1.596 \mathrm{\mu g} \mathrm{g}^{-1}$ de solo (Chagas, 1994). Estes dados reforçam a característica de baixa fertilidade natural, marcante para os solos da área de influência dos reservatórios de Camargos/Itutinga, MG (Siqueira et al., 1995)

O agrupamento das espécies com base nas características ecológicas em pioneiras (aroeira, aroeirinha, jacaré, sabiá e sesbânia) e clímax (guanandi, ipê-amarelo, jatobá e óleo-bálsamo) (Tabela 2), permitiu detectar o comportamento distinto desses grupos em resposta às doses de $P$ aplicadas (Tabela 3). A ausência de interação entre espécies clímax e doses de $\mathrm{P}$, para as variáveis vegetativas, exceção feita à relação raiz:parte aérea, indica que essas espécies tenderam a apresentar o mesmo padrão de resposta em função do aumento na disponibilidade do nutriente. Já no caso das pioneiras, o efeito de doses foi variável conforme a espécie, evidenciado pela interação significativa desses fatores.

Os resultados foram, desta forma, condizentes com a hipótese de que, comparativamente às espécies pioneiras, as classificadas como clímax mostram um crescimento pouco influenciado pelo nível de fertilidade do solo, o que poderia ser indicativo de uma maior adaptação a solos pouco férteis (Marschner, 1991; Lambers \& Poorter, 1992; Siqueira et al., 1995), ou de um rígido ajuste da taxa de crescimento a condições de baixa disponibilidade de nutrientes, o que restringe sua sensibilidade à melhoria nos níveis de fertilidade do solo.

A Fig. 1 demonstra diferenças de produção de matéria seca pelas espécies durante o período de condução do experimento. Observou-se que as espécies pioneiras em comparação às clímax tenderam a apresentar um crescimento inicial mais pronunciado, o que coincide com a maior responsividade do primeiro grupo ao fornecimento de P. Por outro lado, as espécies clímax teriam como mecanismo de adaptação um ajuste da taxa de crescimento, apresentando um desenvolvimento caracteristicamente mais lento.

TABELA 3. Resumo da análise de variância dos dados de crescimento vegetativo, produção de matéria seca de raízes (MSR), parte aérea (MSPA) e total (MST) e relação raiz:parte aérea (RELRP) para os grupos de espécies pioneiras e clímax, submetidas a doses de $P$.

\begin{tabular}{|c|c|c|c|c|c|c|c|}
\hline \multirow[t]{2}{*}{ Fonte de variação } & \multirow[t]{2}{*}{ GL } & \multicolumn{6}{|c|}{ QM } \\
\hline & & Altura & Diâmetro & MSR & MSPA & MST & RELRP \\
\hline Pioneiras & 4 & $2471,6050 * *$ & $161,9595 * *$ & $975,5262 * *$ & $5177,2110^{* * *}$ & $10639,1200 * *$ & $0,0848^{* *}$ \\
\hline Clímax & 3 & $5468,3400 * *$ & $93,6136^{* *}$ & $188,5413^{* *}$ & $1254,5360 * *$ & $2411,7260 * *$ & $0,0376^{* *}$ \\
\hline Pion. x Clím. & 1 & $44596,1370 * *$ & $341,9920 * *$ & $2591,1870 * *$ & $22203,8930 * *$ & $39965,3760 * *$ & $0,1466^{* *}$ \\
\hline Doses de P & 4 & $2009,8990 * *$ & $25,2045^{* *}$ & $101,0266^{* *}$ & $925,6208 * *$ & $1637,0620 * *$ & $0,0281 * *$ \\
\hline Pion. $\mathrm{x}$ Doses & 16 & $518,5803 * *$ & $1,1035^{* *}$ & $6,7549 * *$ & $57,8070 * *$ & $75,9137 * *$ & $0,0149 * *$ \\
\hline Clím. x Doses & 12 & $21,7485^{\mathrm{ns}}$ & $0,2803^{\mathrm{ns}}$ & $0,3015^{\mathrm{ns}}$ & $6,1994^{\mathrm{ns}}$ & $8,0566^{\mathrm{ns}}$ & $0,0065^{* *}$ \\
\hline$(\mathrm{P} \times \mathrm{C}) \times$ Doses & 4 & $1036,9772 * *$ & $13,5995 * *$ & $80,1965 * *$ & $540,6451 * *$ & $1035,3518 * *$ & $0,0111^{* *}$ \\
\hline Tratamentos & 44 & $2082,5760 * *$ & $32,8843^{* *}$ & $179,4433^{* *}$ & $1216,8340^{* *}$ & $2312,6830^{* *}$ & $0,0244 * *$ \\
\hline Resíduo & 180 & 35,8721 & 0,1933 & 0,9948 & 7,1364 & 9,8771 & 0,0026 \\
\hline Total & 224 & & & & & & \\
\hline \multicolumn{2}{|c|}{ Coeficiente de variação (\%) } & 14,22 & 7,49 & 15,61 & 14,79 & 12,85 & 14,45 \\
\hline
\end{tabular}

ns e ** Não-significativo e significativo a $1 \%$ de probabilidade, respectivamente, pelo teste $\mathrm{F}$. 
Estudos (Fetcher et al., 1996; Silva et al., 1997) têm indicado que o desenvolvimento característico da espécie em termos de taxa de crescimento parece ser um fator determinante de sua responsividade à fertilização, cuja rápida incorporação de fotoassi-

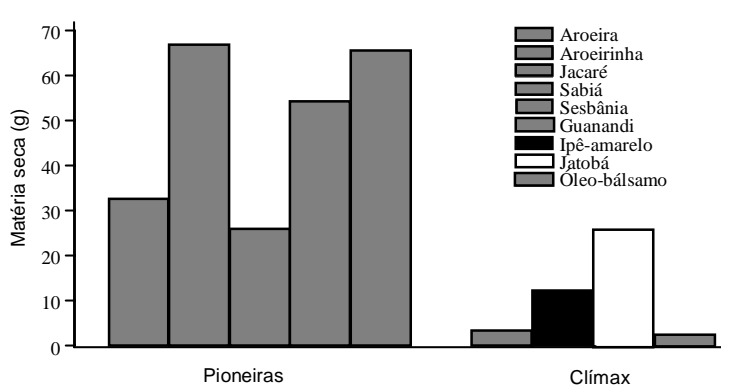

FIG. 1. Matéria seca total máxima observada para espécies florestais pioneiras e clímax em função da fertilização fosfatada. milados e conseqüente incremento na matéria seca numa fase inicial favoreceria o maior requerimento de nutrientes pelas mudas de plantas com crescimento mais agressivo. Os resultados obtidos no presente trabalho confirmaram tal tendência.

\section{Comportamento das espécies pioneiras}

Os modelos matemáticos de melhor ajuste para as variáveis vegetativas das espécies pioneiras em função da aplicação de doses de P (Tabelas 4 e 5), mostram, à exceção da relação raiz:parte aérea de aroeira, aroeirinha e jacaré, respostas positivas das espécies para todas as variáveis analisadas. As estimativas das doses de $\mathrm{P}$ correspondentes a $90 \%$ da produção máxima foram distintas conforme a variável e espécie consideradas, estando associadas ao modelo matemático que explica o tipo de resposta. Assim, as espécies que responderam linearmente mostram que mesmo no maior nível de $\mathrm{P}$ adicionado,

TABELA 4. Equações de regressão entre altura, diâmetro do caule e matéria seca de raízes das espécies pioneiras como variáveis dependentes $(\mathrm{Y})$ das doses de fósforo $(\mathrm{X})$, produção máxima $(\mathrm{PM})$ e dose de fósforo correspondente a $\mathbf{9 0 \%}$ da produção máxima, segundo a equação ajustada.

\begin{tabular}{|c|c|c|c|c|}
\hline Espécie & Equação de regressão & $\begin{array}{c}\text { Produção } \\
\text { máxima (PM) }\end{array}$ & $\begin{array}{c}90 \% \\
\text { da PM }\end{array}$ & $\begin{array}{l}\text { Dose para } 90 \% \\
\text { da PM }\left(\mathrm{mg} \mathrm{dm}^{-3}\right)\end{array}$ \\
\hline & \multicolumn{4}{|c|}{ Altura $(\mathrm{cm})$} \\
\hline Aroeira & $Y=12,2436+2,49089^{* *} X^{0.5}-0,0346924^{* *} X, R^{2}=0,99$ & 56,95 & 51,26 & 533,00 \\
\hline Aroeirinha & $Y=44,4599+0,0568455^{* *} X-0,0000493^{* *} X^{2}, R^{2}=0,99$ & 60,84 & 54,75 & 225,04 \\
\hline Jacaré & $\mathrm{Y}=26,9122+0,148079^{* *} \mathrm{X}-0,0000903^{* *} \mathrm{X}^{2}, \mathrm{R}^{2}=0,99$ & 87,60 & 78,84 & 508,25 \\
\hline Sabiá & $\mathrm{Y}=54,0243+0,0265831^{*} \mathrm{X}-0,0000322^{*} \mathrm{X}^{2}, \mathrm{R}^{2}=0,86$ & 59,51 & 53,56 & 842,45 \\
\hline \multirow[t]{2}{*}{ Sesbânia } & $\mathrm{Y}=60,3829+0,0169488^{* *} \mathrm{X}, \mathrm{R}^{2}=0,93$ & - & 73,94 & 800,00 \\
\hline & \multicolumn{4}{|c|}{ Diâmetro do caule (mm) } \\
\hline Aroeira & $\mathrm{Y}=2,25404+0,0111623^{* *} \mathrm{X}-0,00000749^{* *} \mathrm{X}^{2}, \mathrm{R}^{2}=0,96$ & 6,41 & 5,77 & 452,62 \\
\hline Aroeirinha & $\mathrm{Y}=6,84174+0,211533^{* *} \mathrm{X}^{0.5}-0,00265559^{* * *} \mathrm{X}, \mathrm{R}^{2}=0,99$ & 11,05 & 9,95 & 377,35 \\
\hline Jacaré & $Y=2,50766+0,0068283^{* *} X-0,00000403^{* *} X^{2}, R^{2}=0,99$ & 5,40 & 4,86 & 480,96 \\
\hline Sabiá & $Y=7,16395+0,00253349^{* *} X, R^{2}=0,95$ & - & 9,19 & 800,00 \\
\hline \multirow[t]{2}{*}{ Sesbânia } & $\mathrm{Y}=7,96558+0,00641487^{* *} \mathrm{X}-0,00000473^{* *} \mathrm{X}^{2}, \mathrm{R}^{2}=0,99$ & 10,14 & 9,13 & 215,10 \\
\hline & \multicolumn{4}{|c|}{ Matéria seca de raízes (g) } \\
\hline Aroeira & $\mathrm{Y}=0,656921+0,00704933^{* *} \mathrm{X}, \mathrm{R}^{2}=0,98$ & - & 6,30 & 800,00 \\
\hline Aroeirinha & $\mathrm{Y}=8,52123+0,0258325^{* *} \mathrm{X}-0,0000183^{* *} \mathrm{X}^{2}, \mathrm{R}^{2}=0,96$ & 17,65 & 15,89 & 396,05 \\
\hline Jacaré & $\mathrm{Y}=0,994638+0,00419776^{* *} \mathrm{X}, \mathrm{R}^{2}=0,99$ & - & 4,35 & 800,00 \\
\hline Sabiá & $Y=9,00692+0,0151672^{* *} X-0,00000689^{*} X^{2}, R^{2}=0,89$ & 17,37 & 15,63 & 599,82 \\
\hline Sesbânia & $Y=11,226+0,387381^{* *} X^{0.5}-0,00485797^{*} X, R^{2}=0,98$ & 18,95 & 17,05 & 404,85 \\
\hline
\end{tabular}


a fração que permaneceu disponível para absorção pelas plantas esteve aquém do suprimento ideal para expressão do máximo potencial de crescimento dessas espécies, como no caso da produção de matéria seca de raízes, da parte aérea e total de aroeira e jacaré (Fig. 2).

Em termos percentuais, a produção de matéria seca total de aroeira e jacaré, estimada através das equações (Tabela 5), sofreu sensíveis incrementos em resposta ao fornecimento de P. Já na primeira dose de $\mathrm{P}$ aplicada (100 $\mathrm{mg} \mathrm{dm}^{-3}$ ), o acréscimo de produção em relação à testemunha $\left(0 \mathrm{mg} \mathrm{dm}^{-3}\right)$ foi de $118 \%$ e $74 \%$, respectivamente, o qual persistiu até a dose de $800 \mathrm{mg} \mathrm{dm}^{-3}(941 \%$ e $596 \%$, respectivamente).

Embora menos expressivos, os incrementos na matéria seca até nos maiores níveis de $\mathrm{P}$, também caracterizaram as equações tipo raiz quadrada e quadrática ajustadas para aroeirinha, sabiá e sesbânia
(Fig. 2). Uma elevada resposta a menores doses de $\mathrm{P}$ indica maior viabilidade do uso dessas espécies para recuperação de áreas onde os solos apresentem restrições quanto à disponibilidade do nutriente (Faria, 1993; Lima, 1995).

A partição de fotoassimilados entre raízes e parte aérea, avaliada pelo quociente entre a matéria seca produzida nesses dois componentes da planta, tem sido explorada como indicadora do status nutricional das plantas em relação ao suprimento de $\mathrm{P}$, onde, em geral, à medida que se aumenta a disponibilidade do nutriente no substrato, tem-se um menor crescimento radicular em razão de passar-se a ter a incorporação preferencial dos produtos da fotossíntese nos órgãos aéreos. Dessa forma, o crescimento radicular é favorecido em solos sob condições de deficiência de nutrientes, notadamente de $\mathrm{N}$ e $\mathrm{P}$ (Marschner et al., 1996), e o incremento relativo no órgão de absorção é uma estratégia para extrair o

TABELA 5. Equações de regressão entre matéria seca da parte aérea e total e relação raiz:parte aérea das espécies pioneiras como variáveis dependentes (Y) das doses de fósforo $(\mathrm{X})$, produção máxima (PM) e dose de fósforo correspondente a $90 \%$ da produção máxima, segundo a equação ajustada.

\begin{tabular}{|c|c|c|c|c|}
\hline Espécie & Equação de regressão & $\begin{array}{c}\text { Produção } \\
\text { máxima (PM) }\end{array}$ & $\begin{array}{c}90 \% \\
\text { da PM }\end{array}$ & $\begin{array}{l}\text { Dose para } 90 \% \\
\text { da PM }\left(\mathrm{mg} \mathrm{dm}^{-3}\right)\end{array}$ \\
\hline & \multicolumn{4}{|c|}{ Matéria seca da parte aérea (g) } \\
\hline Aroeira & $Y=2,45579+0,0295594^{* *} X, R^{2}=0,98$ & - & 26,10 & 800,00 \\
\hline Aroeirinha & $Y=20,7499+1,54921^{* *} X^{0.5}-0,0200563^{* *} X, R^{2}=0,99$ & 50,67 & 45,60 & 516,54 \\
\hline Jacaré & $Y=2,7496+0,0236739^{* *} X, R^{2}=0,99$ & - & 21,69 & 800,00 \\
\hline Sabiá & $Y=27,1976+0,707253^{* * *} X^{0.5}-0,0123709^{*} X, R^{2}=0,96$ & 37,31 & 33,58 & 125,88 \\
\hline \multirow[t]{2}{*}{ Sesbânia } & $Y=31,9692+0,0402865^{* * *} X-0,0000263^{* *} X^{2}, R^{2}=0,92$ & 47,40 & 42,66 & 341,56 \\
\hline & \multicolumn{4}{|c|}{ Matéria seca total (g) } \\
\hline Aroeira & $Y=3,11271+0,0366088^{* *} X, R^{2}=0,98$ & - & 32,40 & 800,00 \\
\hline Aroeirinha & $Y=28,4841+2,00838^{* *} X^{0.5}-0,0237747^{* *} X, R^{2}=0,99$ & 70,90 & 63,81 & 623,45 \\
\hline Jacaré & $Y=3,74424+0,0278717^{* *} X, R^{2}=0,99$ & - & 26,04 & 800,00 \\
\hline Sabiá & $\mathrm{Y}=35,3074+1,01047^{* *} \mathrm{X}^{0.5}-0,012549^{*} \mathrm{X}, \mathrm{R}^{2}=0,98$ & 55,65 & 50,08 & 368,74 \\
\hline \multirow[t]{2}{*}{ Sesbânia } & $Y=43,787+0,0611562^{* *} X-0,0000427^{* *} X^{2}, R^{2}=0,94$ & 65,69 & 59,12 & 323,93 \\
\hline & \multicolumn{4}{|c|}{ Relação raiz:parte aérea } \\
\hline Aroeira & $Y=0,377203-0,0143846^{* *} X^{0.5}+0,000345^{* *} X, R^{2}=0,99$ & - & - & - \\
\hline Aroeirinha & $Y=0,378366-0,00367259 X^{0.5}+0,0001237 X, R^{2}=0,44$ & - & - & - \\
\hline Jacaré & $Y=0,378435-0,0113369^{* *} X^{0.5}+0,0001835^{*} X, R^{2}=0,93$ & - & - & - \\
\hline Sabiá & $\mathrm{Y}=0,311465+0,000179354^{* *} \mathrm{X}, \mathrm{R}^{2}=0,84$ & - & - & - \\
\hline Sesbânia & $Y=0,37036+0,0001497 X-0,000000172 X^{2}, R^{2}=0,77$ & - & - & - \\
\hline
\end{tabular}

$*$ e $* *$ Coeficientes significativos a $5 \%$ e $1 \%$, respectivamente. 
máximo do nutriente presente no solo (Clarkson, 1985).

Para as espécies em estudo, o aumento na disponibilidade de $\mathrm{P}$ resultou em alterações diversas em termos de acúmulo de matéria seca no sistema radicular e na parte aérea, produzindo modificações na relação de massa desses compartimentos (RELRP),
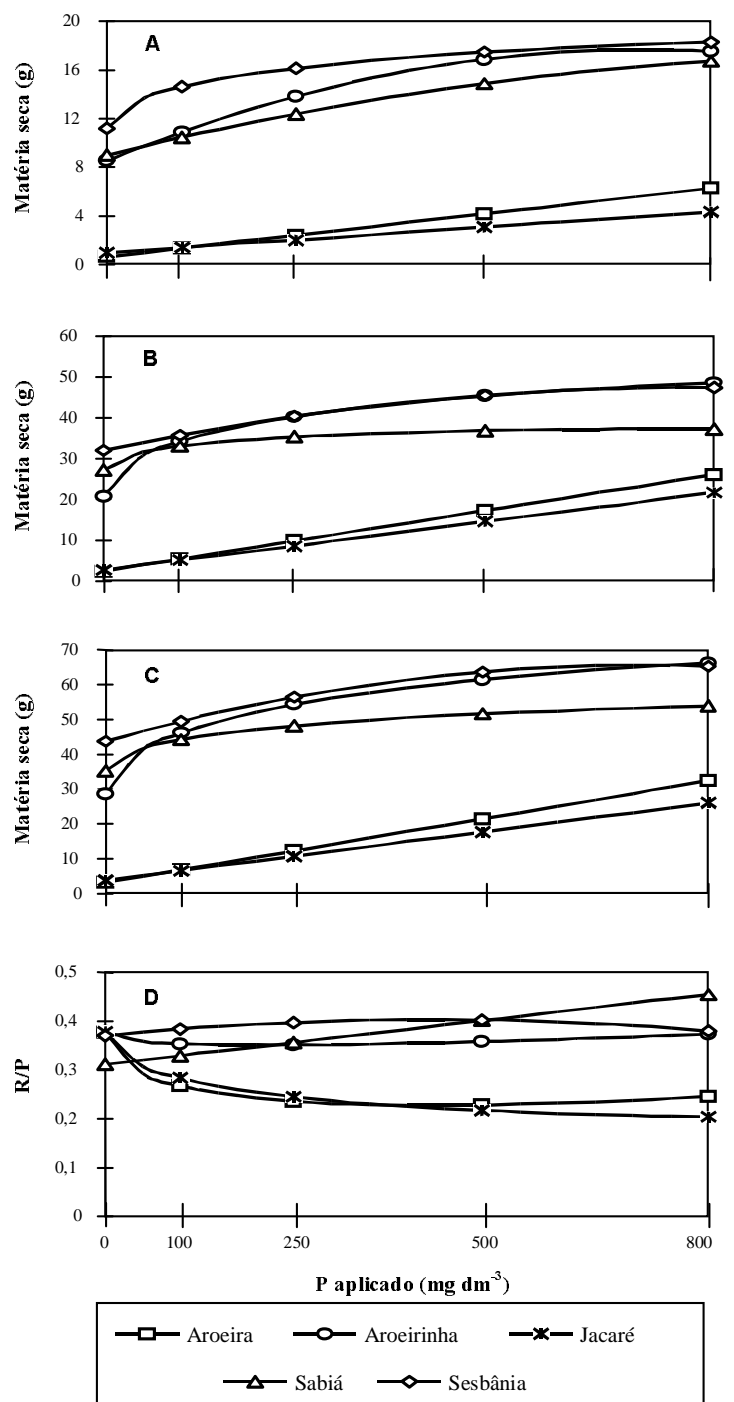

FIG. 2. Efeito da aplicação de fósforo na produção de matéria seca de raízes (A), parte aérea (B) e total $(C)$ e relação raiz:parte aérea - $R / P$ (D) das espécies pioneiras. a qual tendeu a ser decrescente no caso da aroeira, aroeirinha e jacaré, e crescente no caso do sabiá e sesbânia (Fig. 2). A ausência de comportamento padronizado das espécies florestais quanto ao crescimento proporcional de raízes e parte aérea como reação à alterações das condições nutricionais do meio tem-se confirmado em outros trabalhos. Renó et al. (1997) detectaram que a omissão de P provocou aumento significativo na relação para o jacaré, entre outras espécies. Contudo, trabalhos reportam que o fornecimento de $\mathrm{P}$ pode aumentar, reduzir ou não exercer influência sobre a relação raiz:parte aérea (Faria, 1993; Burslem et al., 1995; Rocha, 1995), o que indica que o comportamento pode ser variável em função da espécie florestal envolvida.

As variações, não só em relação ao direcionamento do crescimento mas também quanto ao modelo de equação que explica o comportamento das espécies, reforçam os aspectos ligados às diferenças de flexibilidade no ajuste da relação raiz:parte aérea, em face da modificação das condições nutricionais do ambiente, onde cada espécie pode se comportar de forma peculiar frente ao aumento da disponibilidade de $\mathrm{P}$ no substrato. Neste caso, as espécies adaptadas a ambientes de baixa fertilidade ou que apresentam crescimento mais lento teriam uma menor flexibilidade nesse ajuste (Chapin III, 1980; Clarkson, 1985).

\section{Comportamento das espécies clímax}

$\mathrm{O}$ quociente entre a matéria seca produzida nas raízes e parte aérea (RELRP) foi a única variável vegetativa que mostrou dependência da interação espécies clímax e doses de $\mathrm{P}$ (Tabela 3 ). Quanto às demais variáveis, embora o efeito das doses não tenha se apresentado dependente da espécie, não foi possível o ajuste de equações de regressão que atendam simultaneamente a todas as espécies, uma vez que elas têm padrões diferenciados de crescimento por apresentarem características morfológicas bastante discrepantes.

Esta tendência de resposta é reforçada por outros trabalhos, onde espécies clímax se comportam de forma menos pronunciada em relação à fertilização fosfatada, conduzindo a resultados à primeira vista pouco conclusivos quanto às suas exigências 
nutricionais. Duboc (1994) não encontrou resposta do jatobá ao fornecimento de $\mathrm{P}$ e de outros nutrientes. No estudo a campo de Lima (1995), uma espécie de ipê (Tabebuia crhysotrich) estava entre as menos afetadas pela omissão do $\mathrm{P}$ na adubação de plantio.

Uma reduzida velocidade de crescimento (Fig. 1) parece ser característica inerente às quatro espécies clímax utilizadas no presente estudo. Aliado a isto, comparativamente às pioneiras, o guanandi, ipê-amarelo, jatobá e óleo-bálsamo possuem menor número de sementes por quilograma (Tabela 2), graças ao seu maior tamanho e peso, o que permitiria um maior conteúdo de $\mathrm{P}$ na forma de compostos de reserva acumulado nas sementes e que atenderiam a demanda pelo nutriente numa etapa inicial do crescimento destas espécies (Tisdale et al., 1993; Siqueira et al.,1995, 1998).

Apesar de não terem apresentado respostas em crescimento e produção de matéria seca (Tabela 3 ), as equações de regressão ajustadas para relação raiz:parte aérea (R/P) (Fig. 3) indicam que a partição de fotoassimilados em todas as espécies clímax acarretou um decréscimo do crescimento radicular e conseqüentemente maior acúmulo de matéria seca na parte aérea com o aumento da disponibilidade de P no solo, o que está em conformidade com a tendência geral de redução naquela relação, esperada nessas condições, mas contrariando a hipótese de que espécies de crescimento lento tenham menor flexibilidade de ajuste da relação R/P (Chapin III, 1980; Clarkson, 1985), conforme discutiu-se no tocante às espécies pioneiras.

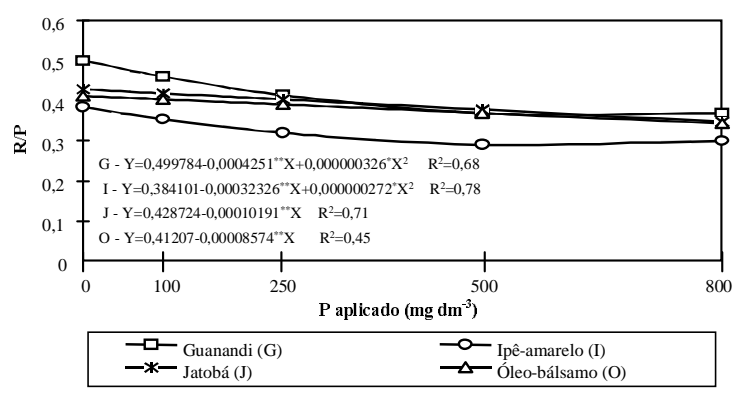

FIG. 3. Efeito da aplicação de fósforo na relação raiz:parte aérea $(R / P)$ das espécies clímax.
Além do grupo ecológico, o requerimento externo de $\mathrm{P}$ pode estar associado a diversos fatores, como o tamanho e conteúdo de nutrientes das sementes, o grau de desenvolvimento do sistema radicular, a dependência micorrízica, a taxa de crescimento e o estádio de desenvolvimento da planta (Marschner, 1991; Kang et al., 1995; Siqueira et al., 1995; Siqueira et al., 1998). Conforme esses autores, maior responsividade ao fornecimento do nutriente deveria ser esperada em espécies de sementes pequenas e com baixos conteúdos de $\mathrm{P}$, com sistema radicular pouco desenvolvido, com maior capacidade micotrófica, maior taxa de crescimento e na fase inicial de desenvolvimento.

Todavia, alguns trabalhos contrariam, em parte, tais premissas. Gonçalves et al. (1992a, 1992b) verificaram que espécies pioneiras, que possuíam sistema radicular mais desenvolvido e raízes finas em maior quantidade, apresentaram as maiores taxas de crescimento e absorveram mais nutrientes que as clímax, nas quais foi constatada a presença de raízes atrofiadas e espessas. No campo, as espécies clímax estudadas por Lima (1995) não apresentaram resposta à adubação com $\mathrm{P}$ aos oito meses após o plantio das mudas, mas na avaliação realizada aos 16 meses foi detectada a resposta tardia dessas espécies. Trabalhando com espécies tolerantes a sombra (não-pioneiras), Burslem et al. (1995) verificaram que não houve resposta em aumento de crescimento para o fornecimento de $\mathrm{P}$, o que foi, em parte, atribuído ao fato de que três das quatro espécies envolvidas no estudo apresentavam micorrizas arbusculares.

Algumas dessas proposições citadas foram comprovadas, enquanto outras não foram sustentadas no presente trabalho e também em outros já mencionados, o que reforça a idéia de que não há modelos abrangentes, e que análises criteriosas se fazem necessárias para que casos fortuitos não sejam tomados como regra. Embora representem propostas que auxiliam o entendimento dos mecanismos que distinguem as espécies quanto às suas exigências nutricionais, há, ainda, a necessidade de estudos mais elaborados com cada espécie, conforme indicam os resultados dos trabalhos realizados até o momento. 
Assim, uma proposta que parece mais condizente com as informações aqui obtidas, é a de que espécies com crescimento lento (clímax), geralmente mais adaptadas a solos com suprimento restrito de nutrientes têm baixa eficiência de utilização, sendo menos responsivas ao seu fornecimento. Por outro lado, as espécies com maiores taxas de crescimento (pioneiras) são mais sensíveis à diminuição da disponibilidade de nutrientes, reduzindo mais drasticamente o seu crescimento, embora mesmo nestas condições ainda apresentem um desenvolvimento mais rápido do que as primeiras (Lambers \& Poorter, 1992).

Com base no exposto, o atendimento dos níveis de suficiência de $\mathrm{P}$ no solo, referentes às espécies pioneiras estudadas, implicaria na aplicação de significativas quantidades de fertilizantes fosfatados e, levando-se em conta as características ecológicas dessas espécies e seu papel no mecanismo da sucessão, a melhor opção de manejo da adubação para este grupo parece ser o fornecimento de uma dose padronizada de $\mathrm{P}$ em formas prontamente solúveis aplicadas na cova de plantio, o que propiciaria o crescimento inicial vigoroso dessas espécies, com uma rápida cobertura da área, protegendo o solo e criando condições de ambiente favoráveis ao estabelecimento das espécies dos grupos sucessionais subsequentes.

Em relação às espécies clímax estudadas, na ausência de respostas às doses de $\mathrm{P}$ e visando racionalizar o uso de insumos, pode-se sugerir, como opção de manejo da fertilização no campo, o fornecimento de uma dose mínima de adubo solúvel, complementada por uma fonte de disponibilidade lenta, como, por exemplo, os fosfatos naturais brasileiros, os quais têm menor custo em relação ao primeiro. A adoção deste manejo torna-se bastante razoável se ponderar que a adubação fosfatada onera a implantação de florestas e além disso pode contribuir para a contaminação do ambiente com íons potencialmente nocivos, como alguns metais pesados que ocorrem como impurezas de determinados fertilizantes fosfatados. As espécies clímax, apresentando crescimento lento, terão à disposição, com o passar do tempo, o P liberado das fontes menos solúveis e, somado a este, o $\mathrm{P}$ advindo do processo de ciclagem da serrapilheira produzida pelas espécies do primeiro grupo sucessional.

\section{CONCLUSÕES}

1. Na fase inicial de crescimento, a responsividade ao fornecimento de P é distinta, de acordo com os grupos sucessionais e com as espécies estudadas.

2. As espécies pioneiras apresentam desenvolvimento mais acentuado que as clímax, respondendo positivamente às doses de $\mathrm{P}$ com incrementos no crescimento.

3. As mudas das espécies clímax são pouco afetadas pelo fornecimento de $\mathrm{P}$, o que caracteriza um baixo requerimento do nutriente pelas mesmas.

\section{REFERÊNCIAS}

BURSLEM, D.F.R.P.; GRUBB, P.J.; TURNER, I.M. Responses to nutrient addition among shade-tolerant tree seedlings of lowland tropical rain forest in Singapore. The Journal of Ecology, Danvers, v.83, n.1, p.113-122, Feb. 1995.

CHAGAS, C. da S. Associação de Latossolo Variação Una e Latossolo Vermelho-Escuro: efeito diferencial da orientação dos estratos de rochas pelíticas pobres. Lavras: ESAL, 1994. 125p. Dissertação de Mestrado.

CHAPIN III, F.S. The mineral nutrition of wild plants. Annual Review of Ecology Systematics, Palo Alto, v.11, p.233-260, 1980.

CLARKSON, D.T. Adaptações morfológicas e fisiológicas das plantas a ambientes de baixa fertilidade. In: SIMPÓSIO SOBRE RECICLAGEM DE NUTRIENTES E AGRICULTURA DE BAIXOS INSUMOS NOS TRÓPICOS, 1984, Ilhéus. Anais. Ilhéus: CEPLAC/SBCS, 1985. p.45-75.

DAVIDE, A.C.; FARIA, J.M.R.; BOTELHO, S.A. Propagação de espécies florestais. Lavras: UFLA, 1995. $41 \mathrm{p}$.

DUBOC, E. Requerimentos nutricionais de espécies florestais nativas: Hymeneaea courbaryl L. var. stilbocarpa (Haynee) Lee et Lang (Jatobá), Copaifera langsdorffii Desf. (Óleo copaíba) e Peltophorum dubium (Spreng) Taub. (Canafístula). Lavras: ESAL, 1994. 68p. Dissertação de Mestrado.

EMBRAPA. Serviço Nacional de Levantamento e Conservação de Solos (Rio de Janeiro, RJ). Manual de métodos de análise de solo. Rio de Janeiro, 1979. $271 \mathrm{p}$. 
FARIA, M.P. Resposta de leguminosas arbóreas ao fósforo e fungo micorrízico em Latossolo Vermelho-Escuro da região Campos das Vertentes (MG). Lavras: ESAL, 1993. 100p. Dissertação de Mestrado.

FARIA, M.P.; SIQUEIRA, J.O.; VALE, F.R.; CURI, N Crescimento inicial da Acácia em resposta a fósforo, nitrogênio, fungo micorrízico e rizóbio. Revista Brasileira de Ciência do Solo, Campinas, v.20, n.2 p.209-216, maio/ago. 1996.

FARIA, M.P.; VALE, F.R.; SIQUEIRA, J.O.; CURI, N Crescimento de leguminosas arbóreas em resposta a fósforo, nitrogênio, fungo micorrízico e rizóbio II. Peltophorum dubium (Spreng.) Taub. Revista Árvore, Viçosa, v.19, n.4, p.433-446, out./dez. 1995.

FETCHER, N.; HAINES, B.L.; CORDERO, R.A.; LODGE, D.J.; WALKER, L.R.; FERNÁNDEZ, D.S.; LAWRENCE, W.T. Responses of tropical plants to nutrients and light on a landslide in Puerto Rico. The Journal of Ecology, Danvers, v.84, n.3, p.331-341, June 1996.

FREIRE, J.C.; RIBEIRO, M.A.V.; BAHIA, V.G.; LOPES, A.S.; AQUINO, L.H. Resposta do milho cultivado em casa de vegetação à níveis de água em solos da região de Lavras-MG. Revista Brasileira de Ciência do Solo, Campinas, v.4, n.5, p.5-8, jan./abr. 1980

GONÇALVES, J.L.M.; FREIXÊDAS, V.M.; KAGEYAMA, P.Y.; GONÇALVES, J.C.; DIAS, J.H. Produção de biomassa e sistema radicular de espécies de diferentes estágios sucessionais. Revista do Instituto Florestal, São Paulo, v.4, Parte 2, p.363-367, mar. 1992a. Edição Especial

GONÇALVES， J.L.M.; KAGEYAMA， P.Y.; FREIXÊDAS, V.M.; GONÇALVES, J.C.; GERES, W.L.A. Capacidade de absorção e eficiência nutricional de algumas espécies arbóreas tropicais. Revista do Instituto Florestal, São Paulo, v.4, Parte 2, p.463-469, mar. 1992b. Edição Especial.

KANG, B.T.; LADIPO, D.O; OFEIMU, O. Phosphorus and liming effects on early growth of selected plant species grown on a Ultisol. Communications in Soil Science and Plant Analysis, Monticello, v.26, n.9/10, p.1659-1673, May 1995

LAMBERS, H.; POORTER, H. Inherent variations in growth rate between higher plants: a search for fisiological causes and ecological consequences.
Advances in Ecological Research, London, v.23, p.188-261, 1992.

LIMA, H.N. Crescimento inicial de sete espécies arbóreas nativas em resposta à adubação com NPK a campo. Lavras: UFLA, 1995. 53p. Dissertação de Mestrado.

LIMA, H.N.; VALE, F.R.; SIQUEIRA, J.O.; CURI, N Crescimento inicial de espécies arbóreas em resposta ao superfosfato simples em campo. Revista da Universidade do Amazonas: Série Ciências Agrárias, Manaus, v.4/5, n.1/2, p.57-69, jan./dez. $1995 / 1996$

MARSCHNER, H. Mechanisms of adaptation of plants to acid soils. Plant and Soil, The Hague, v.134, n.1, p.1-20, July 1991

MARSCHNER, H.; KIRKBY, E.A.; CAKMAK, I. Effect of mineral nutritional status on shoot-root partioniting of photoassimilates and cycling of mineral nutrients. Journal of Experimental Botany, Oxford, v.47, p.1255-1263,Aug. 1996. Special Issue

PARON, M.E.; SIQUEIRA, J.O.; CURI, N.; VALE, F.R. Crescimento da copaíba e guatambu em resposta a fungo micorrízico, superfosfato, nitrogênio e fumigação do solo. Cerne, Lavras, v.2, n.2, p.15-30, jul./dez. 1996

RENÓ, N.B.; SIQUEIRA, J.O.; CURI, N.; VALE, F.R Limitações nutricionais ao crescimento inicial de quatro espécies arbóreas nativas em Latossolo Vermelho-Amarelo. Pesquisa Agropecuária Brasileira, Brasília, v.32, n.1, p.17-25, jan. 1997.

ROCHA, R.C. Desenvolvimento de espécies arbóreas com e sem micorrização, transplantadas para solo degradado contendo doses crescentes de fósforo. Lavras: UFLA, 1995. 74p. Dissertação de Mestrado.

SANCHEZ, P.A.; SALINAS, J.G. Low input technology for managing Oxisols and Ultisols in tropical America. Advances in Agronomy, New York, v.34, p.280406, 1981

SILVA, I.R.; FURTINI NETO, A.E.; CURI, N.; VALE, F.R. Crescimento inicial de quatorze espécies florestais nativas em resposta à adubação potássica. Pesquisa Agropecuária Brasileira, Brasília, v.32, n.2, p.205-212, fev. 1997. 
SIQUEIRA, J.O.; CARNEIRO, M.A.C.; CURI, N.; ROSADO, S.C.S.; DAVIDE, A.C. Mycorrhizal colonization and mycotrophic growth of native woody species as related to successional groups in Southeastern Brazil. Forest Ecology and Management, New York, v.107, p.241-252, 1998.

SIQUEIRA, J.O.; CURI, N.; VALE, F.R.; FERREIRA, M.M.; MOREIRA, F.M.S. Aspectos de solos, nutrição vegetal e microbiologia na implantação de matas ciliares. Belo Horizonte: CEMIG, 1995. 28p

TISDALE, S.L.; NELSON, W.L.; BEATON, J.K.; HAVLIN, J.L. Soil fertility and fertilizers. New York: MacMillan, 1993. 634p.

VETTORI, L. Métodos de análise do solo. Rio de Janeiro: Equipe de Pedologia e Fertilidade do Solo, 1969. 34p. (Boletim técnico, 7). 